\title{
Influence of Rib Stiffness and Limited Long-Term Junction Strength on Geogrid Performance
}

\author{
Werner Müller ${ }^{1}$ (D) $\cdot$ Andreas Wöhlecke ${ }^{2}$ (DD
}

Accepted: 11 September 2019 / Published online: 31 October 2019

(C) The Author(s) 2019

\begin{abstract}
The description of the behavior of geogrids in reinforced soil constructions usually ignores long-term behavior and possible failure modes of junctions between longitudinal and transverse ribs. Two proposals have been made during recent years to overcome this drawback. The first analytic approach applies only to rigid geogrids and assumes a specific tensile-shear failure mode of geogrid junctions. The second numerical approach applies also for non-rigid geogrids and takes into account different modes of junction failure. This note discusses the two approaches focusing on the effects of rib stiffness and limited strength, different failure modes and degradation of junctions. It is shown that the mentioned effects should be considered in geogrid design, because they may alter significantly the long-term geogrid performance.
\end{abstract}

Keywords Geogrid · Junction · Anchor trench · Design rules · Long-term behavior

\section{Introduction}

Forces are transmitted between geogrid reinforcement and soil through two mechanisms (Lopes 2002): 1) interface friction (including possibly adhesion) between soil particles and the surface of the geogrid (GGR); 2) interlocking of soil particles in the openings of the GGR and bearing resistance of the soil. The soil "bears" on the

Electronic supplementary material The online version of this article (https://doi.org/10.1007/s40515-01900095-6) contains supplementary material, which is available to authorized users.

Werner Müller

werner.w.mueller@bam.de; werner.w.mueller@icloud.com

Andreas Wöhlecke

andreas.woehlecke@bam.de

1 Berlin, Germany

2 Division 4.3 Contaminant transport and environmental technologies, Bundesanstalt für Materialforschung und -prüfung (BAM), Unter den Eichen 87, 12205 Berlin, Germany 
transverse rib as it bears along a strip foundation. The interlocking force due to the bearing resistance of the soil is transmitted to the longitudinal ribs via the junctions. The resistance against the pull-out is due to interface friction and bearing resistance of the soil. The extent to which friction and bearing resistance are mobilized along the GGR depends on how stiff the longitudinal ribs are and whether the junction can sustain the loading, Fig. 1, (Sieira et al. 2009; Ziegler and Timmers 2004; Müller 2011; Jacobs et al. 2014, 2016; Bathurst and Ezzein 2016). (In this paper the terms "flexible" and "stiff" or "rigid" refer to the stress-strain behavior of longitudinal ribs and not the flexural rigidity of the entire GGR).

However, mobilization of friction and interlocking force along flexible ribs and limited strength of junctions of GGR are often simplified by tacitly assuming that the GGR is rigid, i.e. displacement is the same everywhere, and that the junctions have unlimited strength while always sustaining the interlocking force (Bräu and Herold 2011). Subsequently, the overall interface frictional force as well as the overall interlocking force are proportional to the shear strength of the soil, anchorage length $L$ and normal stress $\sigma_{n}$ and one obtains the following pull-out resistance $R$ for a GGR embedded into a layer of soil $(\varphi$ : soil friction angle, $c$ : soil cohesion, $\lambda$ : interaction coefficient, $\lambda_{F}$ : interface friction coefficient, $\lambda_{I}$ : interlocking coefficient) (Lopes 2002; Bräu and Herold 2011; Koerner 2012):

$$
R=\lambda(\tan \varphi+c) L \sigma_{n}=\left(\lambda_{F}+\lambda_{I}\right)(\tan \varphi+c) L \sigma_{n}
$$

Swan Jr and Yuan (2016) considered how limited junction strength should be included in the description of GGR behavior. The maximal possible interlocking force for a given GGR and soil was calculated by a theoretical formula, which uses an analogy between a strip foundation and a transverse rib (Lopes 2002). It was compared with experimentally determined tensile-shear strength of the junctions of the GGR. Hence, the approach relies on theoretical estimates of soil-GGR interaction, aligns with the assumption of a completely stiff GGR and neglects effects of the flexibility of longitudinal ribs and degradation of junctions.

Wilson-Fahmy and Koerner (1993), Wilson-Fahmy et al. (1994) already highlighted the importance of limited junction strength and pointed out: "Considering the fact that a great portion of the pull-out force may be transmitted by transverse ribs to the junctions ... the long-term resistance of junctions should be challenged in determining the anchorage capacity of geogrids". Further, in the early 2000s, Ziegler and Timmers (2004) used a numerical discrete element model (DEM) to describe the interaction between the flexibility of the longitudinal ribs and local loading of the junctions. A similar approach was suggested by Bathurst and Ezzein (2016), Ezzein et al. (2015). Based on the model of Ziegler and Timmers (2004), which is equivalent to the incremental load finite element model used by Wilson-Fahmy and Koerner (see Online Resource) (Wilson-Fahmy and Koerner 1993; Wilson-Fahmy et al. 1994), the effects of the flexibility of the longitudinal ribs and the limited junction strength on the anchorage of a GGR were discussed by Müller $(2011,2014)$. Based on the mentioned works, Jacobs et al. (2014, 2016) and Jacobs (2016a) presented a design concept for anchor trenches of the GGR of GGR-reinforced landfill capping systems taking into 


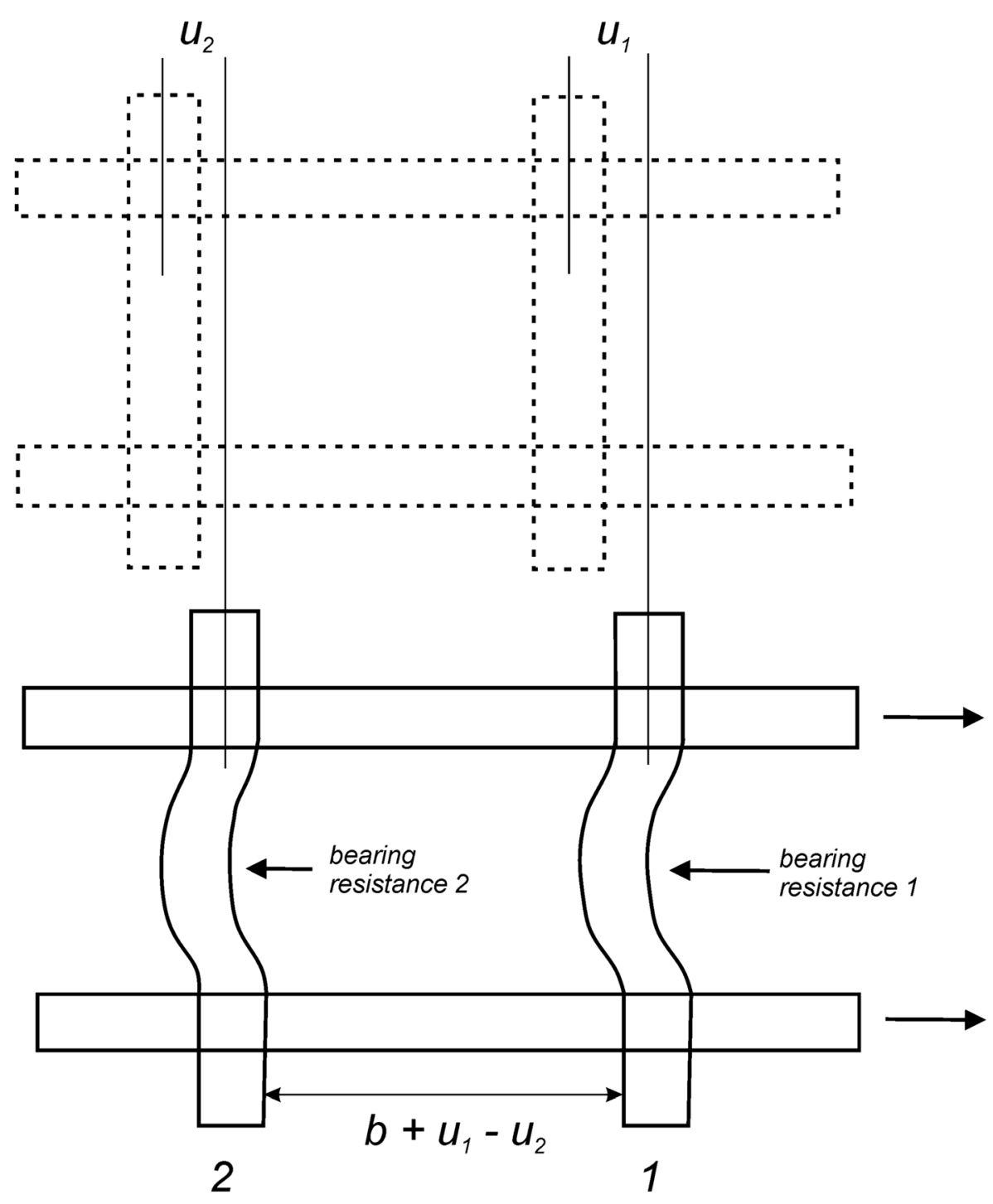

Fig. 1 Schematic view of a GGR before (dotted line) and after (continuous line) a pull-out force acting to the right was applied. Because of the flexibility of the longitudinal ribs, displacement $u_{1}$ of a junction 1 is larger than the displacement $u_{2}$ of the neighboring junction 2 to the left. The length of the opening $b$ of the GGR increases by $u_{1}-u_{2}$. Since mobilization of the bearing resistance of the soil depends on displacement, the interlocking force is larger at junction 1 than at junction 2 . The same applies to the friction force

account GGR flexibility, junction strength and different failure modes of junctions, but did not include the effect of long-term reduction of junction strength.

The paper analyzes the limitations of the concept of Swan Jr and Yuan (2016) and discusses the degradation of junction strength by the aging processes. The model of Jacobs et al. (2014, 2016) and Jacobs (2016a) is briefly introduced, which describes the interplay of the flexibility of longitudinal ribs and loading of junctions, and the handling of the peeling strength of junctions is discussed. Finally, the behavior of 
GGR with limited stiffness and limited junction strength is analyzed referring to Müller (2014). Hence, this paper tries to bring together the different viewpoints. Therefore, more insight can be gained into which specific properties are relevant for an appropriate design of a GGR.

\section{Rigid GGR and Limited Junction Strength - The Swan and Yuan Model}

At the limit state, the transverse rib can plow through the surrounding soil without further increase of the pulling tensile force (as long as the ribs or the junctions do not rupture). Accordingly, Jewell (1996) proposed to estimate the related maximum interlocking force using the soil mechanical theory of the bearing capacity under a strip foundation. The maximum force $F_{1}$, which the transverse rib can exert on a cohesionless soil according to this analogy, is given by ( $d$ : thickness of the transverse rib, $b$ : length of the transverse rib (equal to the width of the opening), $\varphi$ : soil friction angle, $\sigma_{n}$ : normal stress):

$$
F_{1}=b d \sigma_{n} \tan ^{2}\left(\frac{\pi}{4}+\frac{\varphi}{2}\right) e^{\pi \tan \varphi}
$$

The analogy is crude (see Online Resource). However, data from measurements of the pull-out resistance of rigid grids made of steel have shown that Eq. (2) provides an upper limit for the range of the measured values (Jewell 1996).

When a pull-out of the entire GGR is enforced, the entire "package" of gravel or sand particles jammed into the openings of the GGR is displaced. Subsequently, two shear surfaces occur that lie above and below the plane of the GGR in the backfill itself, which Jewell (1996) referred to as fully rough state. If the opening of the GGR has length $a$ and width $b$, the maximum force $F_{2}$, which acts on a transverse rib due to the shear strength of a cohesionless soil, is in the fully rough state ( $\varphi$ : soil friction angle, $\sigma_{n}$ : normal stress) (Jewell 1996):

$$
F_{2}=2 a b \sigma_{n} \tan \varphi
$$

According to Jewell (1996), the increase of the force $F_{1}$ with shear strength of the soil and normal stress should be limited by $F_{2}$ as soon as the interlocking force $F_{1}$ becomes larger than $F_{2}$ and a cross over to a fully rough state becomes more favorable.

Accordingly, using Eq. (2) and (3), Swan Jr and Yuan (2016) suggested complementing Eq. (1) by a limit state equation for junctions (see Fig. 2). For a soil with soil friction angle $\varphi$ and normal stress $\sigma_{n}$, the maximum possible force $F$ which has to be transmitted via each junction of the rigid GGR to the longitudinal ribs is calculated. Then, the additional ultimate limit state equation compares $F$ with the tensile-shear strength of the junction $T_{0}$ as measured in the laboratory by the test ASTM D 7737, method a. 

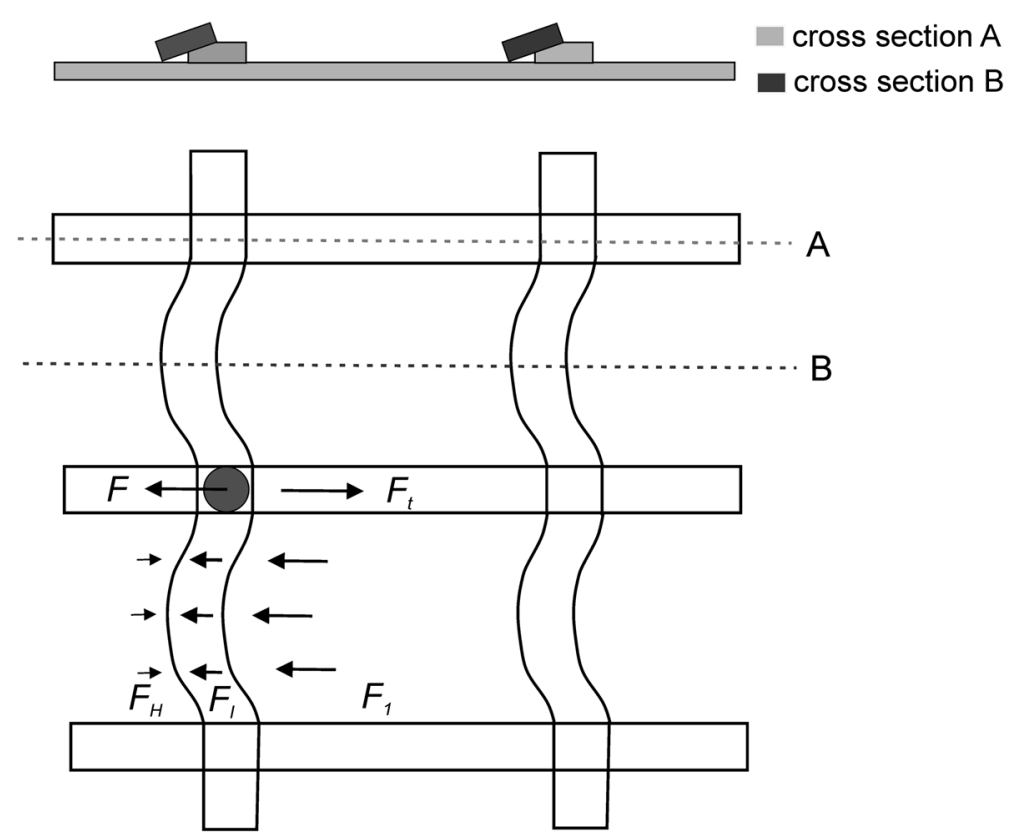

Fig. 2 Lower part: interlocking force $F_{1}$, interface friction force $F_{I}$ and active earth pressure $F_{H}$ act on the transverse rib (Swan Jr and Yuan 2016). The junction is loaded by the $\operatorname{sum} F$ of these forces. $F$ is balanced by the tensile force $F_{t}$ in the longitudinal rib. Tensile shear failure of the junction occurs if this pair of forces exceeds the strength of the junction $T_{0}$. Upper part: schematic view of two cross section of a welded PET GGR (Ziegler and Timmers 2004). Cross section A: before the test. Cross section B: excavated after the pull-out test. The observed rotation (or twisting) of the transverse rib is indicated.

$F$ is the sum of three components:

1. $\quad F_{1}$ and $F_{2}$ are calculated according to Eq. (2) and (3) and the respective smaller force $F_{3}=\min \left(F_{1}, F_{2}\right)$ is used.

2. The interface friction force $F_{I}$ between the GGR and the soil particles is, as usual (Koerner 2012), calculated according to: $F_{I}=2 w b\left(\sigma_{n} \tan \delta+c_{S G}\right)$, where $w$ : width of transverse rib in the longitudinal direction, $b$ : length of the transverse rib (equal to the width of the opening), $\delta$ : soil-GGR interface friction angle, $c_{S G}$ : adhesion at soil-GGR interface, $\sigma_{n}$ : normal stress.

3. On the back side of the transverse rib, in the view of Swan Jr and Yuan (2016), there is an additional pushing force similar to the force that exists due to the active earth pressure exerted by the backfill on a yielding retaining wall $(d$ : thickness of the transverse rib, $b$ : length of the transverse rib, $\varphi$ : soil friction angle, $\sigma_{n}$ : normal stress): $F_{H}=\frac{b d \sigma_{n}}{\tan ^{2}\left(\frac{\pi}{4}+\frac{\varphi}{2}\right)}$.

Hence:

$$
F=F_{3}+F_{I}-F_{H}
$$

For given properties of the cohesionless soil $\varphi, \sigma_{n}$ and GGR properties $a, b, d, w, \delta, c_{S G}$, the ultimate limit state equation with the global safety factor $\gamma$ is: 


$$
T_{0}=\gamma F
$$

The behavior of a rigid GGR with limited junction strength is then described by Eq. (1) and (5).

\section{Limitations and Possible Improvements of the Swan and Yuan Model}

\subsection{Rigid GGR}

The suggestion of Swan Jr and Yuan (2016) is a step forward in handling the long pending problem of how to include long-term junction strength into the assessment and description of the GGR behavior. However, there are limitations. One of which is the assumption of a rigid geogrid. The concept sticks to the theoretical idea of a rigid GGR, because it is assumed that if locally $F_{1}$ becomes as large as $F_{2}$, there is a cross over to a fully rough state and $F_{3}$ is taken to be $F_{2}$ instead of $F_{1}$. However, this assumption only applies if all transverse ribs are loaded in the same way by a pulling tensile force, i.e. in the case of a rigid GGR. Theoretical considerations as well as carefully analyzed experiments have clearly shown that in the case of polymeric GGR this assumption is not valid (Sieira et al. 2009; Ziegler and Timmers 2004; Bathurst and Ezzein 2016; Wilson-Fahmy et al. 1994; Ezzein and Bathurst 2014). The displacement of the transverse ribs and thus the mobilization of the soil resistance is nonlinearly distributed over the length of the embedded GGR. Moreover, part of the length does not even contribute (Section 4 and Fig. 4 ). Hence, $F_{1}$ can become locally larger than $F_{2}$, because the cross over to a fully rough state involving the entire GGR is not possible at once. Therefore, the description of the interplay of flexibility of the longitudinal ribs and loading of junctions needs a completely different approach (Section 4).

The other limitations include the restriction to the short-term values of junction strength and to a specific mode of junction failure. In the following, it is argued how these deficiencies can be overcome to some extent.

\subsection{Including Long-Term Strength and Failure Modes of Junctions}

The bearing resistance of a soil leads to tensile and shear forces within the plane of the junction and possibly a related tensile-shear failure. The strength of the junction $T_{0}$ with respect to this failure mode is used in Eq. (5). It can be measured either by the GRI GG2 test standardized as method $a$ of ASTM D 7737 or method $b$ of ASTM D 7737, which give either the unconfined or confined tensile-shear strength of the junction $T_{0}$ (Koerner 2012; Kupec et al. 2004a, b). However, like the evaluation of the design value of the tensile strength of the longitudinal ribs, the laboratory value of the tensile-shear strength of the junction must be modified by various reduction factors to obtain a relevant value for design. Junctions are the weak points of a GGR considering degradation. This applies to woven, knitted, extruded or welded GGR. Similar to the longitudinal ribs, junctions are subject to aging and various environmental influences (reduction factor $R F_{C H}$ and possibly $R F_{W}$ for weathering effects according to ISO/TR 20432). Additionally, creep can influence junctions (reduction factor $R F_{C R}$ according to ISO/TR 20432). As in the case of ribs, junctions can be damaged during installation 
(reduction factor $R F_{I D}$ according to ISO/TR 20432). Finally, dynamic loads can have an adverse effect on junction strength (this factor is designated by $R F_{D L}$ ). Consequently, if ribs are exposed to all these effects, so are the junctions. Such reduction factors quantify the loss of strength which unavoidably takes place during installation and use. It is therefore necessary to insert a design value $T$ into Eq. (5), which is reduced compared to the test value $T_{0}$ :

$$
T=\frac{T_{0}}{R F_{C R} \times R F_{I D} \times R F_{C H} \times R F_{D L}}
$$

If degradation affects junctions in the same way as ribs, the reduction factors of ribs might be used in Eq. (6). However, there are examples where this is not guaranteed. For example, polyester (PET) material in a weld seam could be pre-damaged by the thermal and mechanical stress during welding causing more rapid hydrolytic aging. Therefore, a test should be undertaken in which the hydrolytic degradation of junction strength is compared with that of tensile strength of the longitudinal rib under the same conditions. Another example includes when junctions fail by mechanisms that do not occur in the ribs. Extruded GGR are often made of partially crystalline polyolefins. The ribs are highly stretched to achieve high strength. Subsequently, molecules and crystallites are strongly oriented, thereby hindering stress cracking. This does not apply to the junction area between the ribs, where poorly oriented material is exposed to stress concentrations. Therefore, checking the stress cracking behavior of junctions under the stress level of the field application is necessary to understand whether stress cracking is an issue during the lifetime of the project. Depending on the failure mechanism, one can conduct ASTM D 7737 tests on aged samples or long-term tensile load tests with sample holders according to ASTM D 7737 on the junctions of the GGR.

In extruded GGR, the transverse rib, the longitudinal rib and the junction lie in the same plane (integral junction). In welded or woven junctions, the transverse and longitudinal ribs lie one above the other. Ziegler and Timmers (2004) demonstrated that in this case the transverse ribs rotate to some extent during the displacement in the soil using steel grids, which incur irreversible plastic deformation during pull-out (Fig. 2). The welded PET GGR excavated after pull-out testing showed similarly deformed transversal ribs. Hence, the bearing resistance of the soil leads not only to tensile and shear forces within the plane of the junction and possibly to a related tensile-shear failure but also to a pair of tensile forces perpendicular to the area of contact between longitudinal and transverse ribs (Fig. 2). These forces try to separate the junction possibly leading to a "peeling failure" (Kupec et al. 2004a, b). Welded seams or adhesive bonded junctions are sensitive to peeling forces. Peeling strength is usually much lower than tensile-shear strength. As such, Jacobs (2016a) observed that the load at which the welded junctions failed in the soil was lower than their confined tensileshear strength, which was attributed to peeling effects. The occurrence of peeling failure depends on the amount of twisting of the transverse rib which increases with the amount of displacement. Failure occurs at a permissible displacement $u_{p, 0}$ and related junction peeling strength $T_{p, 0}$. The limit state equation should not only refer to the long-term value of the tensile-shear strength $T$ but also to the long-term peeling strength $T_{p}$. The peeling behavior can be determined in pull-out tests comparing the 
behavior of specimens, which consists of only longitudinal ribs, and specimens, which consists of longitudinal ribs with one transverse rib, see diagram a in Fig. 3. Details of such a test method are described in Section 3 of (Jacobs 2016a). Using such tests, a criterion for the permissible displacement $u_{p, 0}$ can be established. A junction peeling strength $T_{u, 0}$, which can be inserted into Eq. (6) to give a long-term peel strength $T_{u}$, is related to the permissible displacement at which failure occurs.

\subsection{Consequences of the Suggested Improvements for GGR Performance}

The suggested improvements can be illustrated by modifying the example given by Swan Jr and Yuan (2016) accordingly: a GGR in a supporting layer $\left(\varphi=37^{\circ}\right)$ under a roadway. With $\sigma_{n}=77 \mathrm{kPa}$ from traffic load, they got from Eq. (2): $F_{1}=169 \mathrm{~N}$. A fully
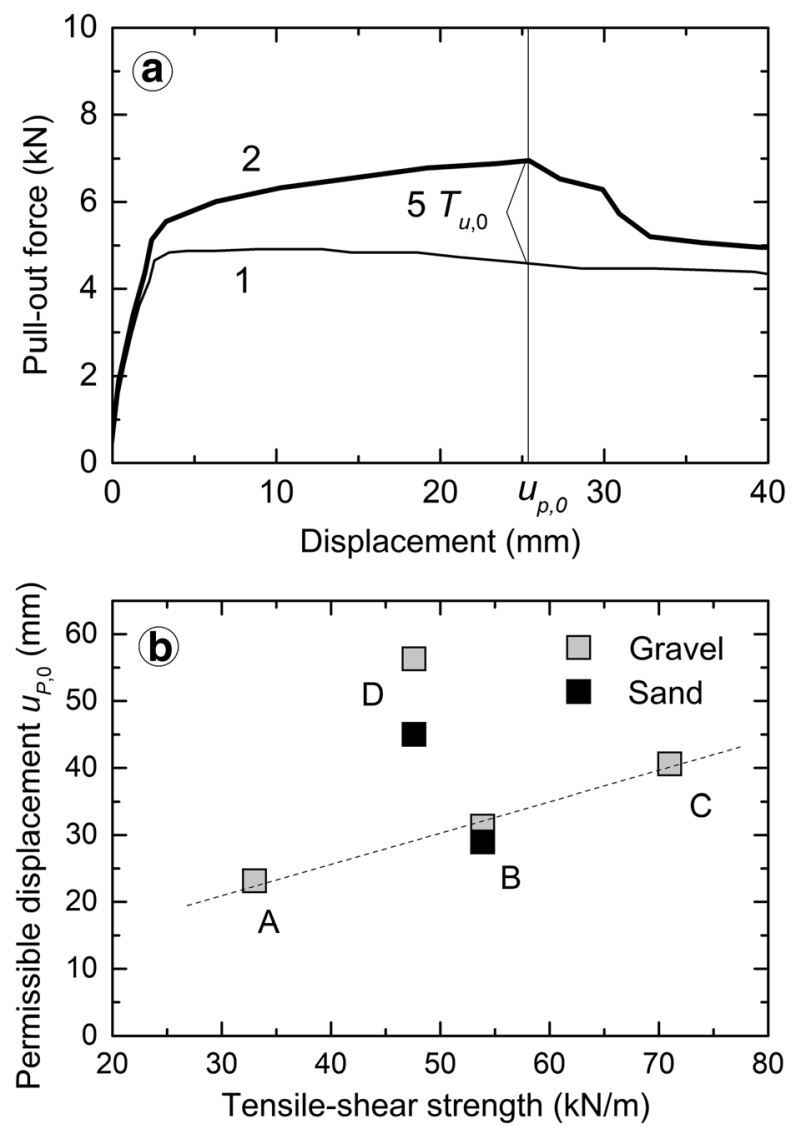

Fig. 3 Diagram a: Curve 1 shows pull-out force versus displacement of a test with GGR specimen consisting of five longitudinal but no transverse ribs. Curve 2 shows pull-out force versus displacement with GGR specimen consisting of five longitudinal and one transverse rib. The difference between these curves gives the loading function of the junction. The peeling failure occurred at a permissible displacement $u_{p, 0}$ and peeling strength $T_{u, 0}$. Data taken from (Jacobs 2016a). Diagram b: The displacement at which junctions failed (permissible displacement $u_{p, 0}$ ) in a small-scale pull-out test (sand $0 / 2$ and gravel 0/32) as a function of initial tensile-shear strength of the junction (Jacobs 2016a). Variants A, B, C and D of the welded GGR were investigated by Jacobs (2016a) 
rough state, on the other hand, gave via Eq. (3): $F_{2}=75 \mathrm{~N}$. The interface friction $F_{I}$ of the chosen GGR product and the active earth pressure $F_{H}$ provided only neglectable contributions. Therefore, $F=75 \mathrm{~N}$. Laboratory tensile-shear strength was $T_{0}=245 \mathrm{~N}$. The global safety was then $\gamma=3.2$.

According to the arguments above, this calculation should be modified as follows: the interlocking force can locally become significantly greater than $75 \mathrm{~N}$ since the tensile force is unevenly distributed along the embedded GGR. On the other hand, junction strength is reduced by degradation, creep, etc. Simply assuming that the reduction factors of the junctions are similar to those of the longitudinal ribs, reduction factors of PET geogrids of $R F_{C R}=1.5, R F_{I D}=1.1$ and $R F_{C H}=1.2$ result from investigation in connection with the BAM certification (BAM 2013), yielding an overall reduction factor of about 2 . Therefore, an overall reduction factor of junctions of at least two and even significantly greater is not unrealistic. Assuming that such an overall factor applies to the GGR in the above example, the factor of safety would only be $\gamma=$ 1 at a value of $F=120 \mathrm{~N}$. Since the soil fails at $169 \mathrm{~N}$, the long-term strength of junctions might locally become smaller than maximum interlocking force. The occurrence of this situation depends on the flexibility of the GGR.

The main argument for not considering junction strength is that the manufacturers produce their GGR in such a way that the short-term strength of junctions is much higher than their averaged loading. In the light of this discussion, this argument is questionable. Long-term strength of the junction might very well be a relevant material resistance concerning the behavior of the GGR in the long run depending on degradation and mode of failure.

\section{Flexible GGR with Limited Junction Strength}

\subsection{The Flexible GGR Model}

To take into account the complex interaction between junction strength, flexibility of longitudinal ribs and soil strength, characteristic functions derived from small scale GGR pull-out and shear tests are used (Jacobs et al. 2014, 2016; Jacobs 2016a). These functions describe the mobilization of the friction force, tensile force in the longitudinal ribs and loading of the junctions as function of local GGR displacement. Local GGR displacements and pull-out forces are numerically calculated using a kind of DEM which can be shown to be equivalent to the finite element model of WilsonFahmy and Koerner (1993), Ziegler and Timmers (2004), Müller (2011, 2014) (see Online Resource for the description of the model). The DEM was adapted by Jacobs (2016a) to the various geometric conditions in an anchor trench (e.g. including deviation force at the top and toe).

Figures 3 and 4 show examples of the mentioned mobilization functions: 1) the interface friction force versus displacement curve $\tau_{s G}(u)$ top right diagram b in Fig. 4 (Müller 2014; Jacobs 2016a); 2) loading of the junction (interlocking force) versus displacement (bottom right diagram f in Fig. 4 and diagram a in Fig. 3 (Müller 2014; Jacobs 2016a); 3) friction force versus displacement for the sliding trench failure, where the GGR together with the overlying soil layer slides on the underlying soil layer. Such a function is necessary for studying the failure modes of trenches. A series 

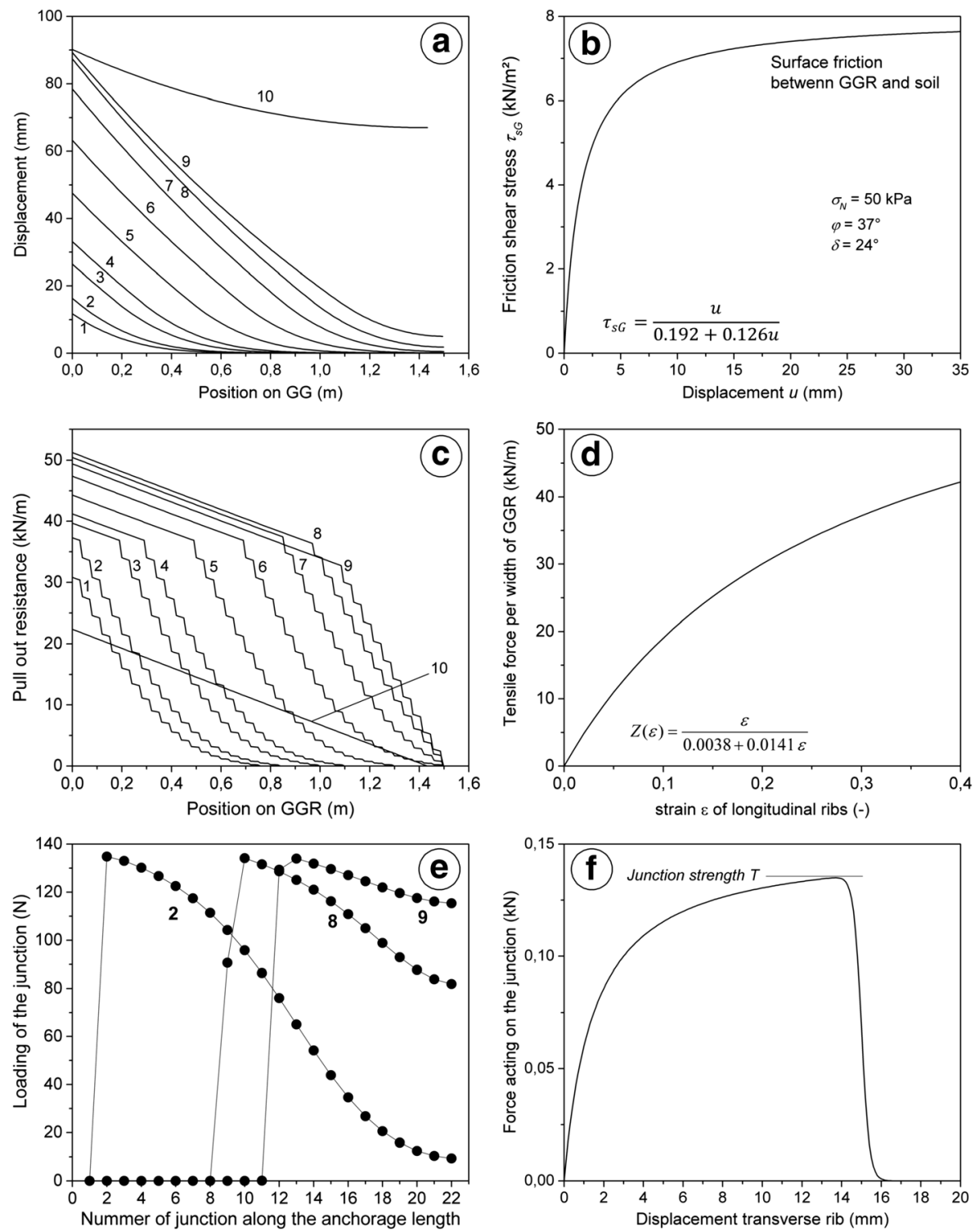

Fig. 4 Diagrams on the right show the mobilization functions: Diagram b: example of a function of friction shear stress $\tau_{s G}(u)$ versus displacement $u$. Diagram d: example of a tensile force versus strain diagram $Z(\varepsilon)$. The hyperbolic curves interpolate data, which are typically observed for extruded polypropylene geogrids (Sieira et al. 2009). Diagram f: interlocking force, which is transferred per junction from the loaded transversal into the longitudinal rib, as function of the displacement $u$ and cut-off value for the limited junction strength $T$. This hypothetical curve serves as a representative example for the mechanical loading of a junction with degraded strength $T$. Diagrams on the left show the behavior of a flexible GGR with degraded junction strength. The simulation of the pull-out process proceeded stepwise by increasing the displacement by a small increment. Steps of the simulation are shown in diagram a, $c$ and $f$ indicated through numbers. Diagram a: the distribution of the pull-out resistances over the geogrid length during a pull-out test. Diagram c: the distribution of displacements. Diagram e: the loading of the junctions during the pull-out. The figure is taken from (Müller 2014) 
of all three functions must be determined because of the dependence of the functions on soil properties and normal stress; 4) tensile force versus elongation for the longitudinal ribs (middle right diagram d of the force-strain-curve $Z(\varepsilon)$ in Fig. 4 (Müller 2014; Jacobs 2016a).

The interlocking force, which the junction can sustain, is either limited by its tensileshear strength or a critical displacement and related peel strength (bottom right diagram f in Fig. 4 and diagram a in Fig. 3 (Müller 2014; Jacobs 2016a). Therefore, the effect of the limited strength of the junctions is included in the DEM by limiting the range and domain of the function No. 2 (Müller 2014; Jacobs 2016a) and a cut-off parameter for range and domain of this function expresses the failure of junctions. Moreover, extending beyond a cut-off-value indicates the numerical simulation that interlocking no longer contributes to the interaction of soil and GGR. Similarly, long-term tensile strength of the longitudinal ribs is included by a cut-off parameter for the possible range of tensile forces described by the stress-strain-function No. 4 (Müller 2014; Jacobs 2016a).

Jacobs (2016a) determined the respective functions and cut-off parameters for various types of a welded GGR by many small-scale pull-out and shear tests in sand and gravel at normal stresses of 20, 50 and $100 \mathrm{kN} / \mathrm{m}^{2}$. Diagram a in Fig. 3 shows an example (one transverse rib, sand $0 / 2,100 \mathrm{kN} / \mathrm{m}^{2}$ ).

The model was validated by comparing results from large scale experimental test of GGR in anchor trenches (overall embedded length up to $2.1 \mathrm{~m}$ (Girard et al. 2006)) and field measurements of the displacement of a GGR in the anchor trench of a landfill capping system during and after installation (Jacobs et al. 2014, 2016; Jacobs 2016a) with model calculation using the mobilization functions described above. Agreement between measured and calculated pull-out resistance in the first case and measured and calculated displacement along the embedded GGR in the second case was found.

Peeling failure of welded junctions occurs at a certain displacement depending on soil and GGR properties. Therefore, in addition to a permissible tensile-shear strength a permissible displacement $u_{p, 0}$ of the junctions was introduced (Jacobs 2016a), Fig. 3, diagram a. Jacobs et al. (2014, 2016), Jacobs (2016a) used such a permissible displacement in the simulation instead of a peeling strength. Then, the permissible displacement, which is short-term, has to be reduced in view of the long-term behavior of junction strength as discussed in Section 3. There exists a relation between the permissible displacement, which leads to peeling failure in a pull-out test, and the initial tensile-shear strength of the junction, as indicated in the diagram $\mathrm{b}$ in Fig. 3. $u_{p, 0}$ was determined for products with the same type of welded junctions (A, B, C) but increasing short-term tensile-shear strength. $u_{p, 0}$ increases linear with the strength (Jacobs 2016a). If a long-term value for the tensile-shear strength is established (Eq. (6)), an associated long-term cut-off parameter for the permissible displacement can be extrapolated from this relation. Alternatively, long-term values of the critical displacement can be derived by applying the test and analysis of the test results shown in Fig. 3 to specimen, which, however, have been properly aged. Reliable cut-off values for long-term tensile-shear strength and long-term permissible displacement must be implemented into the flexible GGR model to get a full assessment of the repercussions of junction failure. 


\subsection{Behavior of a GGR with Flexible Longitudinal Ribs and Large Junction Strength in an Anchor Trench}

For the special case of anchor trenches of the GGR in a reinforced landfill capping system (i.e. one layer of GGR embedded in a layer of soil) with various geometries of the anchor trenches as well as embedded lengths, normal stresses and tensile loads, the pull-out resistances were calculated by Jacobs (2016a) in a comprehensive parameter study using the flexible GGR model and compared with the determination of the pullout resistances according to a conventional design method. Junction strength was assumed to be larger than maximum interlocking force. The two types of failure modes of the anchorage (pull-out and sliding) were included by comparing the balance of the forces evaluated at each state of loading for each mode and switching accordingly from one mode to the other (details are given in (Jacobs 2016a), Section 5.2.1.1, p. 123). The deviation forces were included by a position dependent normal stress above and below the GGR (Jacobs 2016b). Based on this parameter study, a criterium was established for this special case, by which one can decide whether a conventional design of a trench is safe or not.

A linear relation between pull-out force $R$ and the parameters $\tan \varphi, \sigma_{n}$ or $L$ (Eq. (1)) is only valid over a small range of parametric values. The interaction coefficient $\lambda$ depends on the chosen range and it is difficult to determine it experimentally. When $\lambda$ is determined in small-scale pull-out (ASTM 6706) or pull-through tests (DIN 60009) with a small box, results for $R$ might be incorrect when Eq. (1) is applied to long embedded lengths. This is because the ratio of the length which is really mobilized (for a given soil and normal stress) to the overall embedded length will become smaller by increasing the overall length and decreasing the stiffness of the ribs and the share of friction force on the pull-out resistance.

The stiffness of the longitudinal ribs can be quantified by the averaged long-term tensile stiffness $J_{m, \infty}[\mathrm{kN} / \mathrm{m}]$, which is determined according to (Jacobs 2016a) as follows: stress-behavior of a GGR depends on temperature and deformation velocity. From creep testing stress-strain-curves for various temperatures and velocities may be determined (isochronous curves). Taking the stress-strain curve for the temperature of application and the velocity related to the lifetime of the construction and using a linear approximation, the slope of the stress-strain-line gives the relevant averaged tensile stiffness. The division of this quantity by the reduction factors for the tensile strength of the longitudinal ribs (see Section 3) gives $J_{m, \infty}$. The share of the friction force is the ratio $\rho_{m d}=\lambda_{F} / \lambda$ [dimensionless] as determined in small-scale pull-out tests. The anchorage length is designated $L[\mathrm{~m}]$. Accordingly, Jacobs et al. (2014, 2016), Jacobs (2016a) introduced a parameter called stiffness of the anchorage $k_{R}\left[\mathrm{kN} / \mathrm{m}^{2}\right]$, which depends on the quantities just defined according to the following Eq. 7:

$$
k_{R}=\frac{J_{m, \infty} \rho_{m d}}{L}
$$

The anchorage resistance is the tensile force, which the anchored GGR can sustain. If for a given anchor trench the ratio $r$ of the anchorage resistance (as calculated by the numerical model) to the anchorage resistance (as calculated by a conventional 
approach) is $\geq 1$, then the conventional approach is considered to be safe compared to the numerical simulation. If $r<1$, then it is unsafe. Assuming an interaction coefficient $\lambda$ as determined in small-scale laboratory pull-out test, Jacobs (2016a) determined the anchorage resistance and $k_{R}$ for the many different cases of anchor trench geometries and loading conditions. Hence, the reliability of the conventional method was checked for the given GGR varying these conditions (Jacobs 2016a) and expressed in terms of $k_{R}$. Fig. 6.22 in (Jacobs 2016a) shows the results of the study: for each of the many cases studied, $r$ was plotted as function of the respective anchor trench stiffness $k_{R}$. In various cases, the conventional approach was not safe, while being safe in many. As expected, it was in the cases of small $k_{R}$, that the conventional approach did not correctly represent the behavior of the GGR, i.e. $r<1$. For the studied GGR with its specific stiffness and share of friction, it was found that in all cases the conventional approach was safe $(r \geq 1)$ for an anchorage stiffness greater than $1820 \mathrm{kN} / \mathrm{m}^{2}$ i.e. $r$ was greater independent of the variation of the geometry of the anchorage trench, the slope angle and the normal stress. On the other hand, a conventionally calculated anchorage resistance had to be reduced by an additional reduction factor of 1.67 to avoid problems in cases where the anchorages stiffness was $<1820 \mathrm{kN} / \mathrm{m}^{2}$, i.e. the variation of geometry of the anchorage trench, slope angle and normal stress gave in many such cases values of $r$ smaller than one and in some cases even as small as 0.6.

Sliding of the GGR was the relevant mode of failure in almost all simulated cases which occurred after the mobilization of friction and bearing resistance due to an intimate contact between soil and GGR. Jacobs (2016a) concentrated on the difference due to the flexibility of the longitudinal ribs and the geometry of the anchor trench and used the short-term values of the cut-off parameters of tensile-shear strength and permissible displacement of the junctions. If strength of junction is larger than maximum interlocking force, failure of junctions is not an issue. However, even in this case, a conventional design based on the rigid GGR model can considerably underestimate the pull-out (or anchorage) resistance of a layer of a flexible, interlocking dominated GGR embedded into a layer of soil.

\subsection{The Behavior of a GGR with Flexible Longitudinal Ribs and Degraded Junction Strength}

Diagram A, C and D in Fig. 4 shows the result of the simulation of a GGR by the DEM with flexible longitudinal ribs and limited junction strength, which is pulled out of a layer of soil (Müller 2014). The same numerical flexible GGR model as in Section 4.2 was applied, however, with different mobilization function and cut-off values as describe in diagram b, d, f of Fig. 4 to simulate a pull-out test. While Jacobs (2016a) analyzed a PET-GGR, the functions in diagram $b, d$, f are characteristic for a polypropylene-GGR. (Sieira et al. 2009). If the flexible longitudinal ribs of the entrenched GGR are loaded by a tensile force, the displacements of the transverse ribs are not evenly distributed (diagram a in Fig. 4). The tensile force is greatest in the front area of the embedded length and decreases along the longitudinal ribs because the tensile force continuously decreases due to the already mobilized interface friction and interlocking forces (diagram $\mathrm{c}$ in Fig. 4). From a certain critical length onward, there is no (or only a very small) displacement and no friction force and no interlocking force is mobilized. At a certain value of the tensile force, either the soil or the junctions in the 
front area of the embedded length fail by internal shear failure or junction rupture. The soil fails once its bearing capacity is reached. The junctions rupture, if they cannot sustain the forces, which must be transferred from transverse to longitudinal ribs at or below the bearing capacity of the soil. The failure of soil or junction must happen at first in the front area because displacement and accordingly mobilized bearing resistance is the largest. If the tensile force is further increased the "zone" of soil failure or junction rupture will move along the GGR (diagram e in Fig. 4). The process resembles the opening of a zipper. Once the end is reached, pull-out of the entire GGR will begin. Hence, the maximum tensile force (pull-out force) occurs in between a succession of local limit states. The assumption inherent to the conventional approach, that the pullout force would increase linearly with the embedded length, is therefore not applicable (or only an approximation of unknown accuracy) for a flexible interlocking dominant GGR. The strength of the junction in the long run is as important for the behavior of the GGR as that of the longitudinal ribs.

Failure of degraded and overloaded junctions does not necessarily lead to a full-scale failure of the anchorage. The consequences of junction failure depend on the flexibility of the longitudinal ribs and the overall embedded length. This may be understood as follows: a tensile force leads to a certain configuration of displacements and forces along the mobilized part of the GGR. Let the GGR be subjected to a tensile force in such a way that the junctions in the front area of the embedded length are loaded at the limit of their strength or permissible displacement (simulation step 2 in diagrams a, c, e in Fig. 4). If the strength or permissible displacement of the junctions is reduced due to degradation, the zipper mechanism will start. However, the interface friction of the intact longitudinal ribs remains mobilized. Hence, the tensile force, which drives the zipper mechanism, is continuously reduced due to the friction of longitudinal ribs. If there is still a large enough mobilizable part of the GGR at the end of the embedded length, one gets a configuration along this intact and mobilizable part, which is equivalent to an original configuration, however, at a lower tensile force. If this reduction in tensile force fits the reduced strength or permissible displacement, the zipper will stop. If not, the zipper will run through and pull-out for the entire GGR will occur. This leads to the basic question for values of long-term tensile-shear strength and long-term permissible displacement of the GGR junctions the zipper condition can be prevented. This can only be decided on the basis of the experimental study of long-term junction strength, as described in Sections 3 and 4 and the simulation of GGR performance in Section 4.

\section{Conclusions}

The strength of GGR junctions is limited and degrades over the long term. GGR junctions degrade at least as much as GGR ribs. A safe design must consider this effect. For this purpose, experimental based long-term tensile shear strength values of junctions as well as for the stiffness longitudinal ribs should be taken into account. The tensile-shear-strength of a junction can be determined using method $a$ or method $b$ of ASTM D7737. Small-scale pull-out tests on a GGR-specimen with only one transverse rib can be used to check whether other failure modes are relevant. The peeling strength of junctions can be indirectly characterized by such pull-out-tests in terms of a 
permissible displacement of transverse ribs. These tests can be applied to properly aged specimens to determine the long-term tensile-shear strength and/or the long-term permissible displacement. In special cases, it might be necessary to use long-term tensile shear testing or long-term small-scale pull-out testing to assess the effect. To account for the flexibility of the longitudinal ribs of the GGR and therefore the distribution and quantity of the forces acting on the junctions a numerical model combined with experimentally determined mobilization functions and cut-off values can be used to evaluate the factor of safety with respect to the long-term junction strength. The mobilization functions describe interaction between GGR and soil.

\section{Compliance with Ethical Standards}

Conflict of Interest On behalf of all authors, the corresponding author states that there is no conflict of interest.

Open Access This article is distributed under the terms of the Creative Commons Attribution 4.0 International License (http://creativecommons.org/licenses/by/4.0/), which permits unrestricted use, distribution, and reproduction in any medium, provided you give appropriate credit to the original author(s) and the source, provide a link to the Creative Commons license, and indicate if changes were made.

\section{References}

BAM: Zulassung für Bewehrungsgitter aus Kunststoff für Deponieoberflächenabdichtungen. Amts- und Mitteilungsblatt. 43, 16-38 (2013). https:/opus4.kobv.de/opus4-bam/frontdoor/index/index/docId/8. Accessed 30 Aug 2019

Bathurst, R., Ezzein, F.: Geogrid pullout load-strain behaviour and modelling using a transparent granular soil. Geosynth. Int. 23, 271-286 (2016). https://doi.org/10.1680/jgein.15.00051

Bräu, G., Herold, C.: Recommendations for Design and Analysis of Earth Structures Using Geosynthetic Reinforcements - EBGEO. Ernst \& Sohn, Berlin (2011)

Ezzein, F.M., Bathurst, R.J.: A new approach to evaluate soil-geosynthetic interaction using a novel pullout test apparatus and transparent granular soil. Geotext. Geomembr. 42, 246-255 (2014). https://doi. org/10.1016/j.geotexmem.2014.04.003

Ezzein, F.M., Bathurst, R.J., Kongkitkul, W.: Nonlinear load-strain modeling of polypropylene geogrids during constant rate of strain loading. Polym. Eng. Sci. 55, 1617-1627 (2015). https://doi.org/10.1002 /pen.23999

Girard, H., Briançon, L., Rey, E.: Experimental tests for geosynthetic anchorage trenches. In: Kuwano, J., Kosegi, J. (eds.) Proceedings of the eighth International Conference on Geosynthetics, pp. 29-36. Millpress, Rotterdam (2006)

Jacobs F. Interaktionsmodell zur Bemessung von Verankerungsgräben mit Geogittern. Dissertation, RWTH Aachen University (2016a) https://d-nb.info/1130327353/34. Accessed 04 Sept 2019

Jacobs, F. Interaction model for design of geogrid pullout. In: Proceedings of 6th European Geosynthetics Congress, Ljubljana, Slovenia, pp.794-803 (2016b)

Jacobs, F., Ziegler, M., Vollmert, L., Ehrenberg, H.: Explicit design of geogrids with a nonlinear interface model. In: Ziegler, M., Bräu, G., Heerten, G., Laackmann, K. (eds.) Proceedings of the 10th International Conference on Geosynthetics. German Geotechnical Society (DGGT), Essen (2014)

Jacobs, F., Ziegler, M., Vollmert, L., Ehrenberg, H.: Design of geogrid pullout with a nonlinear interaction model. In: Kelsey, C. (ed.) GeoAmericas 2016 - 3rd Pan-American Conference on Geosynthetics. Minerva - Technology, Resources, and Information, Jupiter (2016)

Jewell, R.A.: Soil Reinforcement with Geotextiles. Thomas Telford, London (1996)

Koerner R.M. Designing with Geosynthetics, 6th edn. Xlibris Corporation (2012), http://bookstore.xlibris. com. Accessed 04 Sept 2019 
Kupec J McGown A. Ruiken A. Index testing of the junction strength of geogrids. In: Proceedings of the Third Asian Regional Conference on Geosynthetics, Now and Future of Geosynthetics in Civil Engineering, Seoul, pp. 797-802 (2004a)

Kupec, J., McGown, A., Ruiken, A.: Junction strength testing for geogrids. In: Floss, R., Bräu, G., Nußbaumer, M., Laackmann, K. (eds.) Proceedings of the Third European Geosynthetics Conference, Geotechnical Engineering with Geosynthetics. Deutsche Gesellschaft für Geotechnik (DGGT) Und Technische Universität München, pp. 717-722. TUM-ZG, München (2004b)

Lopes, M.L.: Soil-geosynthetic interaction. In: Shukla, S.K. (ed.) Geosynthetics and their Applications. Thomas Telford, London (2002)

Müller, W.W.: Zur Bemessung der Verankerung von Bewehrungsgittern aus Kunststoff beim Schutz von Böschungen vor hangparallelem Gleiten. Bautechnik. 88, 347-361 (2011). https://doi.org/10.1002 /bate.201101472

Müller, W.W.: Long-term pull-out resistance and materials properties of geogrids. In: Ziegler, M., Bräu, G., Heerten, G., Laackmann, K. (eds.) Proceedings of the 10th International Conference on Geosynthetics. German Geotechnical Society (DGGT), Essen (2014)

Sieira, A.C., Gerscovich, D., Sayão, A.: Displacement and load transfer mechanisms of geogrids under pullout condition. Geotext. Geomembr. 27, 241-253 (2009). https://doi.org/10.1016/j.geotexmem.2008.11.012

Swan Jr R.H. Yuan Z.A. Theoretical analysis of the maximum load transferred to the junctions of a geogrid confined in granular soil. In: Proceedings of the Geo-Chicago 2016: Forging a Path to Bona Fide Engineering Materials. ASCE Library, pp. 37-48 (2016)

Wilson-Fahmy, R.F., Koerner, R.M.: Finite element modelling of soil-geogrid interaction with application to the behavior of geogrids in a pullout loading condition. Geotext. Geomembr. 12, 479-501 (1993). https://doi.org/10.1016/0266-1144(93)90023-H

Wilson-Fahmy, R.F., Koerner, R.M., Sansone, L.J.: Experimental behavior of polymeric geogrids in pullout. J. Geotech. Eng. 120, 661-667 (1994). https://doi.org/10.1061/(ASCE)0733-9410(1994)120:4(661)

Ziegler, M., Timmers, V.: A new approach to design geogrid reinforcement. In: Floss, R., Bräu, G., Nußbaumer, M., Laackmann, K. (eds.) Proceedings of the Third European Geosynthetics Conference, Geotechnical Engineering with Geosynthetics. Deutsche Gesellschaft für Geotechnik (DGGT) and Technische Universität München, pp. 661-667. TUM-ZG, München (2004)

Publisher's Note Springer Nature remains neutral with regard to jurisdictional claims in published maps and institutional affiliations. 